\title{
Adenosine Triphosphate-dependent Taurocholate Transport in Human Liver Plasma Membranes
}

Henk Wolters, Folkert Kuipers, Maarten J. H. Slooff, * and Roel J. Vonk

Department of Pediatrics, University of Groningen, and * Department of Surgery, University Hospital, $9712 \mathrm{KZ}$ Groningen, The Netherlands

\begin{abstract}
Transport systems involved in uptake and biliary secretion of bile salts have been extensively studied in rat liver; however, little is known about these systems in the human liver. In this study, we investigated taurocholate (TC) transport in canalicular and basolateral plasma membrane vesicles isolated from 15 human livers (donor age 6-64 yr). ATP stimulated the uptake of TC into both canalicular and basolateral human liver plasma membrane vesicles (cLPM and blLPM, respectively). Considerable interindividual variations in the transport velocity were observed in the different membrane preparations used: $9.0 \pm 1.3$ (mean $\pm \mathrm{SEM}, n=17$; range 1.6-18.0) and 9.3 \pm 2.0 (range 1.129.8) $\mathrm{pmol} \mathrm{TC} \cdot \mathrm{mg} \mathrm{protein}^{-1} \cdot \mathrm{min}^{-1}$ at $1.0 \mu \mathrm{M} \mathrm{TC}$ for CLPM and blLPM, respectively. TC transport was temperature sensitive and showed saturation kinetics with a high affinity for TC $\left(K_{\mathrm{m}} 4.2 \pm 0.7 \mu \mathrm{M}\right.$ and $3.7 \pm 0.5 \mu \mathrm{M}$ for cLPM and blLPM, respectively). Transport was dependent on the ATP concentration and saturable $\left(K_{\mathrm{m}} 0.25 \pm 0.03 \mathrm{mM}, n=3\right)$. Neither nitrate, which reduces membrane potential, nor the protonophore FCCP strongly inhibited ATP-dependent TC transport, indicating that membrane potential and proton gradient are not involved in this process. TC transport was significantly inhibited by the classical anion transport inhibitor $4,4^{\prime}$-diisothiocyanostilbene-2,2'-disulfonate $(250 \mu \mathrm{M})$ and the glutathione conjugate $S$-(2,4-dinitrophenyl) glutathione $(100 \mu \mathrm{M})$. In conclusion, high affinity ATP-dependent TC transport is present in human liver at both the canalicular and the basolateral sides of the hepatocyte. (J. Clin. Invest. 1992. 90:2321-2326.) Key words: canalicular plasma membrane vesicles - basolateral plasma membrane vesicles - primary active transport • DNP-SG
\end{abstract}

\section{Introduction}

Transport systems involved in vectorial hepatic transport of bile salts (i.e., uptake at the sinusoidal side and secretion at the canalicular pole of the hepatocyte) have been studied extensively in rat liver. However, little is known about these processes in the human liver. Vesicles are the system of choice to study these processes, since no interference by intracellular metabolism occurs and the media inside and outside the vesicles

Address reprint requests to Dr. H. Wolters, Department of Pediatrics, University of Groningen, Bloemsingel 10,9712 KZ Groningen, The Netherlands.

Received for publication 10 February 1992 and in revised form 1 May 1992.

J. Clin. Invest.

(c) The American Society for Clinical Investigation, Inc.

$0021-9738 / 92 / 12 / 2321 / 06 \$ 2.00$

Volume 90, December 1992, 2321-2326 can be defined exactly and manipulated easily. We have recently developed a method to isolate canalicular ( CLPM $)^{1}$ and basolateral (blLPM) plasma membrane fractions simultaneously from human liver (1), which enables us to study transport processes in both types of membranes of the same liver.

Studies using membrane vesicles from rat liver indicate that uptake of taurocholate (TC) from the blood into the hepatocyte at the basolateral (sinusoidal) membrane is sodium dependent (2-6). Only a single study is known to us in which TC transport was investigated in human liver membranes: in basolateral membrane preparations isolated from two human livers, sodium-dependent TC transport could also be demonstrated (7).

The excretion of TC into bile has been shown to be sodium independent and membrane potential dependent in rat liver $(5,8,9)$. A protein of $\sim 100 \mathrm{kD}$ appears to be involved in this transport process (10-12). The magnitude of the membrane potential, however, is too small to explain the actual bile salt concentration gradients that exist over the canalicular membrane (5). Recently, primary ATP-dependent TC transport was demonstrated in rat canalicular membranes (13-17), which apparently acts synergistically with potential-dependent TC transport (18). Photoaffinity labeling and reconstitution studies revealed that a $110-\mathrm{kD}$ glycoprotein functions as carrier in the ATP-dependent TC transport (16).

Besides the bile salt transport system, the existence of two other ATP-dependent transporters has been demonstrated in rat canalicular membranes, namely, a transporter for non-bile acid organic anions, e.g., $S$-(2,4-dinitrophenyl)glutathione (DNP-SG), cysteinyl leukotrienes and glucuronides (19-25), and probably bile acid sulfates (25), and the P-glycoprotein, the product of the multidrug-resistance gene, which transports mainly hydrophobic cations (26). Available data indicate that the multidrug-resistance transporter is different from the anion carriers. There is also evidence from in vitro studies that TC transport and non-bile acid organic anion transport proceed via different systems $(15,16)$. This has previously been inferred from the existence of the Dubin-Johnson syndrome in man (27), and mutant Corriedale sheep (28) and Wistar rats $(29,30)$, all showing impaired secretion of non-bile salt organic anions with normal bile salt secretion.

Since bile salt secretion is an important determinant of bile flow, information on mechanisms of hepatic bile salt transport in human liver may provide better insight into normal bile

1. Abbreviations used in this paper: blLPM, basolateral domains of liver plasma membranes; cLPM, canalicular domains of liver plasma membranes; DIDS, 4,4'-diisothiocyanostilbene-2,2'-disulfonic acid; DNP-SG, S-(2,4-dinitrophenyl)glutathione; FCCP, carbonylcyanide$p$-trifluoromethoxyphenylhydrazone; LAP, leucine aminopeptidase; RE, relative enrichment of enzyme activities; TC, taurocholate; UW, University of Wisconsin. 
formation in man, and improve our understanding of mechanisms involved in the etiology of cholestasis. We therefore investigated TC transport in human liver plasma membranes. In this work we demonstrate the presence of an ATP-dependent TC transport system in human liver plasma membranes.

\section{Methods}

Materials. $\left[{ }^{3} \mathrm{H}\right] \mathrm{TC}$ was purchased from DuPont/New England Nuclear (Boston, MA), FCCP (carbonylcyanide- $p$-trifluoromethoxyphenyl-hydrazone) was from Fluka AG (Buchs, Switzerland), and valinomycin and DIDS (4,4'-diisothiocyanostilbene-2,2'-disulfonic acid) were purchased from Sigma Chemical Co. (St. Louis, MO). DNP-SG was kindly provided by Dr. P. Zimniak (Division of Gastroenterology, University of Arkansas for Medical Sciences, Little Rock, AR). All other chemicals used were of analytical grade.

Human livers. Human liver tissue was obtained from livers harvested from multiorgan donors (six female and nine male, age 6-64 $y r)$. Consent from legal authorities and family was obtained for the explantation of organs for transplantation purposes. The donor livers were split in order to be able to perform reduced-size liver transplants in children. The liver tissue remaining after the splitting procedure was used for the isolation of plasma membranes. Some livers discarded for transplantation after harvesting were used as well. Livers were perfused with University of Wisconsin (UW) solution (31) and stored in this buffer at $4^{\circ} \mathrm{C}$ until the start of the isolation procedure, which was performed within $48 \mathrm{~h}$.

Isolation and characterization of plasma membrane fractions. CLPM and blLPM were isolated and characterized as described previously (1), with a small modification: $0.1 \mathrm{mM}$ PMSF, a protease inhibitor, was added to the homogenization buffer. Membrane vesicles were stored in liquid nitrogen from 4 to 21 mo until use. No indication of loss of transport activity was observed during storage under these conditions. Marker enzymes, $\mathrm{Na}^{+} \mathrm{K}^{+}$-ATPase (32) for basolateral and leucine aminopeptidase (33) for canalicular membranes, were determined as described. Protein was determined by the method of Lowry et al. (34) using BSA (Sigma Chemical Co.) as standard. Enrichments (specific enzyme activity of the membrane preparation relative to the specific enzyme activity in the total homogenate) of the canalicular membrane preparations were $59 \pm 8$ for leucine aminopeptidase and $21 \pm 5$ for $\mathrm{Na}^{+} \mathrm{K}^{+}$-ATPase, and for basolateral preparations $14 \pm 2$ and $38 \pm 6$, respectively ( 17 preparations). We have previously shown that the subcellular organelles endoplasmic reticulum, mitochondria, Golgi membranes, and lysosomes, are only slightly enriched, if at all, in our membrane preparations (1).

Transport studies. TC transport was measured using a rapid filtration technique. Experiments were performed at $37^{\circ} \mathrm{C}$ in a final volume of $100 \mu \mathrm{l}$. Unless specified otherwise, the incubation medium contained various concentrations of $\left[{ }^{3} \mathrm{H}\right] \mathrm{TC}, 1.0 \mathrm{mM}$ ATP, an ATP regenerating system $(10 \mathrm{mM}$ creatine phosphate and $0.1 \mathrm{mg} / \mathrm{ml}$ creatine kinase), $1.0 \mathrm{mM}$ ouabain, and $0.5 \mathrm{mg} / \mathrm{ml} \mathrm{BSA}$ in $10 \mathrm{mM} \mathrm{Tris} \cdot \mathrm{HCl} /$ $0.25 \mathrm{M}$ sucrose/ $10 \mathrm{mM} \mathrm{MgSO} / 4 / 0.2 \mathrm{mM} \mathrm{CaCl}_{2}, \mathrm{pH} 7.4$; final membrane protein concentration was $0.2 \mathrm{mg}$ protein $\cdot \mathrm{ml}^{-1}$. Similar incubations without ATP served as blanks. Transport was stopped by adding $2.5 \mathrm{ml}$ ice-cold buffer ( $10 \mathrm{mM}$ Tris $\cdot \mathrm{HCl} / 0.25 \mathrm{M}$ sucrose $/ 10 \mathrm{mM}$ $\mathrm{MgSO}_{4} / 0.2 \mathrm{mM} \mathrm{CaCl}_{2}, \mathrm{pH} 7.4$ ). Vesicles were filtered through 0.45 $\mu \mathrm{m}$ mixed (nitrate/acetate) cellulose filters (Millipore HAWP; Millipore Corp., Bedford MA), which were prefiltered with $1 \mathrm{ml} 1.0 \mathrm{mM}$ TC and washed twice with $2.5 \mathrm{ml}$ buffer. Radioactivity on the filter was measured in an Wallac liquid scintillation counter (LKB, Turku, Finland). Experiments were performed in triplicate, with at least three different membrane preparations.

$K_{\mathrm{m}}$ and $V_{\max }$ values were calculated using double reciprocal plots of $1 / v$ as a function of $1 /$ [substrate]; the intercept on the $\mathrm{x}$-axis reflects $-1 / K_{\mathrm{m}}$, the intercept on the $\mathrm{y}$-axis $1 / V_{\max }$.

Values are expressed as mean \pm SEM unless indicated otherwise. In some studies least-squares linear regression analysis was applied.

\section{Results}

ATP-dependent TC transport. TC uptake into human liver plasma membrane vesicles at $37^{\circ} \mathrm{C}$ was stimulated by ATP (Fig. 1). Large differences in transport rate between different membrane preparations were observed ( see below), but qualitatively the different membrane preparations showed the same time dependence: initial, rapid uptake was followed by slower uptake, probably a consequence of simultaneous leakage of TC out of the vesicles according to the TC gradient produced. Finally, probably because the ATP concentration in the system was reduced, release of TC occurred. This release was slower in the blLPM fraction shown in Fig. 1 than in the cLPM fraction, probably because in the latter fraction more Mg-ATPase was present (1), leading to faster consumption of ATP. No ATPdependent transport of TC was observed when experiments were performed on ice (data not shown). To verify that uptake and not ATP-dependent binding was measured, studies were performed in the presence of different extravesicular concentrations of sucrose. With increasing osmolarity, less TC was taken up in the presence of ATP (Fig. 2), indicating that only a small fraction of the measured uptake reflects binding to the membranes. Binding of TC to the membrane vesicles (association at infinite medium osmolarity) amounted to 8 and $12 \%$ of the total uptake in normal medium in two different cLPM preparations, and to 8 and $9 \%$, respectively, for blLPM fractions isolated from the same livers. Thus, the contribution of binding was similar for both types of membranes.

Localization of ATP-dependent TC transport. ATP-dependent TC transport was observed in both canalicular and basolateral membrane fractions. Large interindividual differences were observed: initial rates $1.6-18.0 \mathrm{pmol} \mathrm{TC} \cdot \mathrm{mg}^{-1} \cdot \mathrm{min}^{-1}$ for CLPM and 1.1-29.8 for blLPM (medium concentration, $1.0 \mu \mathrm{M}$ TC). In some of the preparations the transport rate (on protein basis) in basolateral membranes was higher than in the canalicular fraction, whereas in others this ratio was opposite (see below). Mean TC transport rate was $9.0 \pm 1.3$

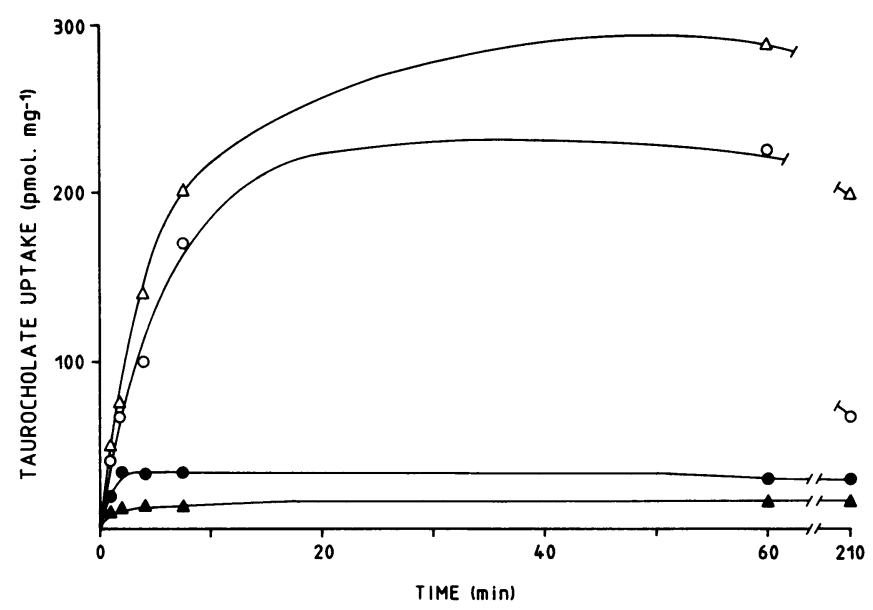

Figure 1. TC uptake into human CLPM and blLPM: effect of ATP. Association of TC (medium concentration $1.0 \mu \mathrm{M}$ ) with membrane vesicles was determined at $37^{\circ} \mathrm{C}$, in the absence (closed symbols) or presence (open symbols) of $1.0 \mathrm{mM}$ ATP. ०, cLPM; $\triangle$, blLPM. Data are from a representative experiment (see text). The experiment was performed in triplicate; SD are not shown for clarity, they were $<15 \%$ of the mean values. 


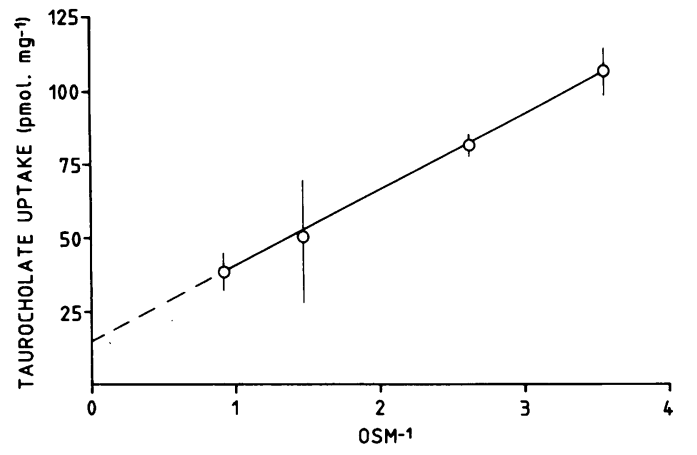

Figure 2. Effect of medium osmolarity on TC uptake in human liver plasma membrane vesicles. ATP-dependent uptake was determined after $30 \mathrm{~min}$ incubation in medium ( TC concentration $1.0 \mu \mathrm{M}$ ) with different concentrations of sucrose. Data $( \pm S D)$ are from a typical experiment in triplicate. Similar observations were made in four other membrane preparations. By extrapolation to the abscissa an estimate of the amount of TC bound to the membranes can be made.

$\mathrm{pmol} \cdot \mathrm{mg}^{-1} \cdot \mathrm{min}^{-1}$ for $\mathrm{cLPM}$ and $9.3 \pm 2.0 \mathrm{pmol} \cdot \mathrm{mg}^{-1}$. $\mathrm{min}^{-1}$ in blLPM ( 17 membrane preparations isolated from 15 different livers). It is theoretically possible that ATP-dependent TC transport is present only in human canalicular membranes, as described for rat liver $(14,15)$. Due to unavoidable contamination with canalicular membranes, ATP-dependent TC transport would in this case also be measurable in the blLPM fraction. This possibility could, however, be excluded. The relative amounts of cLPM present in the cLPM and blLPM fractions can be quantified by measuring the relative enrichment ( $R E$ ) in leucine aminopeptidase (LAP) activity. If ATP-dependent TC transport were performed by canalicular membranes only, the transport rates corrected for the presence of cLPM (by dividing by LAP enrichment) should be identical in CLPM and blLPM. Transport rates thus calculated, however, yielded values of $0.19 \pm 0.04 \mathrm{pmol} \cdot \mathrm{min}^{-1} \cdot \mathrm{mg} \mathrm{prot}^{-1}$. RE $(\text { LAP })^{-1}$ for cLPM and $0.69 \pm 0.10$ for blLPM preparations $(P<0.001)$, indicating that the ATP-dependent TC transport is not only located on the canalicular, but also on the basolateral side of the membrane. Similarly, to rule out the possibility that transport is located on blLPM only, transport rates were corrected for the presence of blLPM by dividing by the RE for $\mathrm{Na}^{+} \mathrm{K}^{+}$-ATPase. Mean velocities were $0.56 \pm 0.08$ for canalicular and $0.27 \pm 0.05 \mathrm{pmol} \cdot \mathrm{min}^{-1} \cdot \mathrm{mg} \operatorname{prot}^{-1} \cdot \mathrm{RE}\left(\mathrm{Na}^{+} \mathrm{K}^{+}\right.$ATPase $)^{-1}$ for basolateral membrane preparations $(P<0.01)$. These data thus show that the ATP-dependent TC transport activity is present in both blLPM and cLPM.

Age dependency of ATP-dependent TC transport. There seemed to be a tendency for a decrease in transport rate with donor age (Fig. 3). However, it appeared that the purity of the membrane preparations was also age dependent to some extent: with increasing age, less enrichment with $\mathrm{Na}^{+} \mathrm{K}^{+}$-ATPase was observed in both cLPM and blLPM. Enrichments in LAP activity were not age dependent. Thus, it was essential to correct the uptake rate for the purity of the plasma membrane preparation. When this was done for the quantity of blLPM by dividing the transport rate by the enrichment of $\mathrm{Na}^{+} \mathrm{K}^{+}$-ATPase activity in the membranes (Fig. 4), there was a tendency for a decrease in uptake rate with age for the basolateral preparation only. Transport rate corrected for LAP showed a decrease with age for both CLPM and blLPM, probably because of the

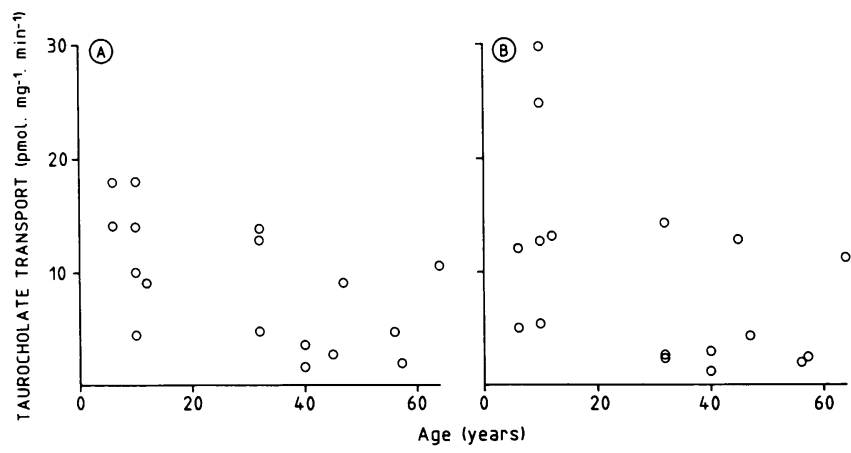

Figure 3. Age dependency of ATP-dependent TC transport in human liver membranes. Initial rapid TC uptake in medium containing 1.0 $\mu \mathrm{M}$ TC was determined as described in Methods. $(A)$ cLPM $(r$ $=-0.567 ; P<0.01) ;(B)$ blLPM $(r=-0.479 ; P<0.05)$.

occurrence of relatively less basolateral membranes in preparations of the older donors, as mentioned earlier.

No correlations between ATP-dependent TC transport and donor gender, preservation time of the liver in UW solution, or storage time of the membranes in liquid nitrogen were observed.

Kinetics of ATP-dependent TC transport. Transport of TC, measured in the presence of $1.0 \mathrm{mM} \mathrm{ATP}$, showed saturation kinetics. Fig. 5 gives an example of the relationship between TC concentration and transport rate. The apparent $K_{\mathrm{m}}$ for TC was $4.2 \pm 0.7 \mu \mathrm{M}$ for cLPM (four preparations), which is similar to the $K_{\mathrm{m}}$ determined for blLPM $(3.7 \pm 0.5 \mu \mathrm{M}$; five preparations). As stated above, large differences in transport rates were observed for different preparations used. $V_{\max }$ values ranged from 6.5 to $188 \mathrm{pmol} \cdot \mathrm{mg} \mathrm{prot}^{-1} \cdot \mathrm{min}^{-1}$ (five basolateral and four canalicular preparations isolated from six different livers).

Fig. 6 shows the dependence of transport rate on ATP concentration in a typical experiment. Transport was saturable; the apparent $K_{\mathrm{m}}$ for ATP was $0.25 \pm 0.03 \mathrm{mM}(n=3) . V_{\max }$ for the three preparations (isolated from three different livers) studied ranged from 4.3 to $21 \mathrm{pmol} \cdot \mathrm{mg}^{-1} \cdot \mathrm{min}^{-1}$.

Role of membrane potential and $\mathrm{pH}$ gradients. To further characterize ATP-dependent TC transport, we studied the effects of several compounds. Since blLPM and cLPM showed

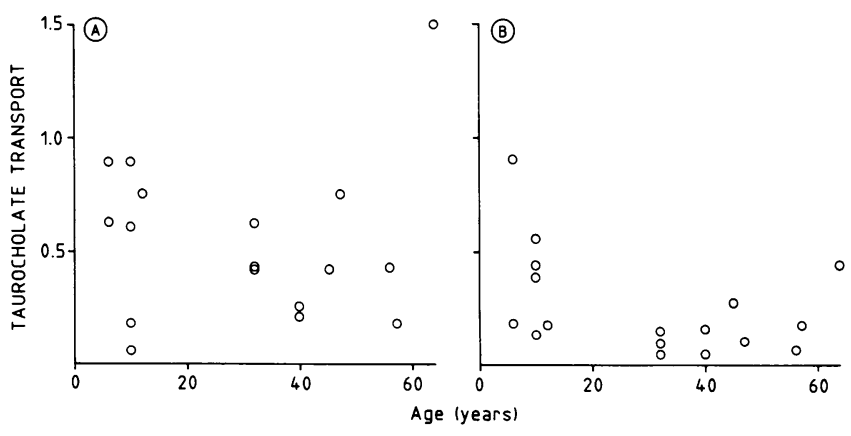

Figure 4. Age dependency of ATP-dependent TC transport in human liver membranes. Transport rates, as presented in Fig. 3, were corrected for the amount of basolateral membranes actually present in the preparations by dividing the transport rate $\left(\mathrm{pmol} \cdot \mathrm{mg}^{-1} \cdot \mathrm{min}^{-1}\right.$ ) by the relative enrichment of the basolateral marker enzyme $\mathrm{Na}^{+} \mathrm{K}^{+}$-ATPase. $(A)$ cLPM $(r=0.069)$; $(B)$ blLPM $(r=-0.409)$. No significant correlation with age was observed. 


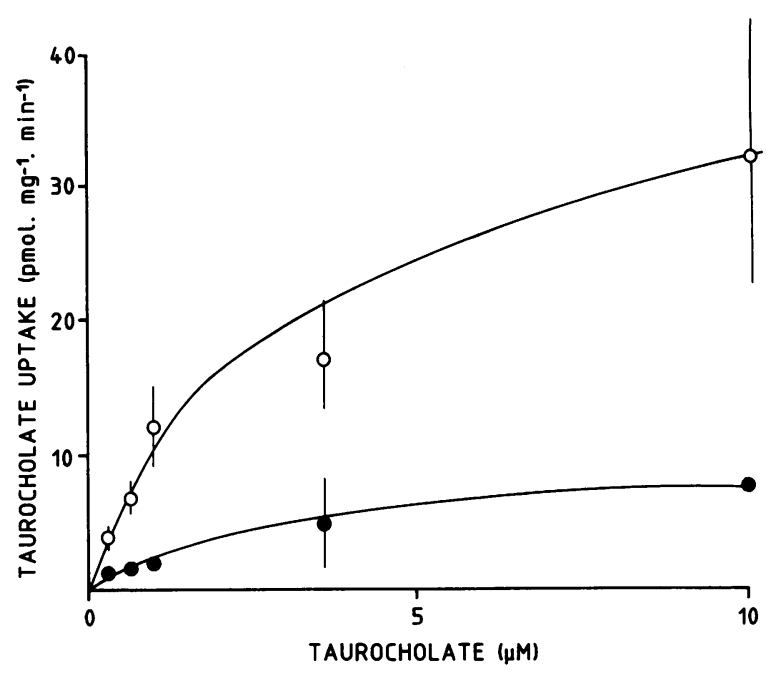

Figure 5. Effect of TC concentration on ATP-dependent TC transport in human liver plasma membrane vesicles. Initial rapid uptake was determined at different TC concentrations in the presence of $1.0 \mathrm{mM}$ ATP; uptake in the absence of ATP served as blanks. Values are mean \pm SD of an experiment in triplicate. ๑, cLPM; O, blLPM. Qualitatively similar curves were observed in seven additional membrane preparations (see text).

similar $K_{\mathrm{m}}$ values for TC uptake, it is highly likely that the same transporter is responsible for transport in both vesicle preparations. We therefore did not study effects of the various compounds on CLPM and bILPM separately, but all studies were done at least once with a preparation of each membrane type. No qualitatively different effects on CLPM and blLPM were observed.

Initial rates of ATP-dependent TC transport were not dependent on $\mathrm{pH}$ gradient or membrane potential. The protonophore FCCP, which dissipates $\mathrm{pH}$ gradients, only slightly inhibited transport. The presence of nitrate, a permeable anion that dissipates a possible membrane potential, stimulated transport (Table I). The $\mathrm{K}^{+}$ionophore valinomycin, which collapses

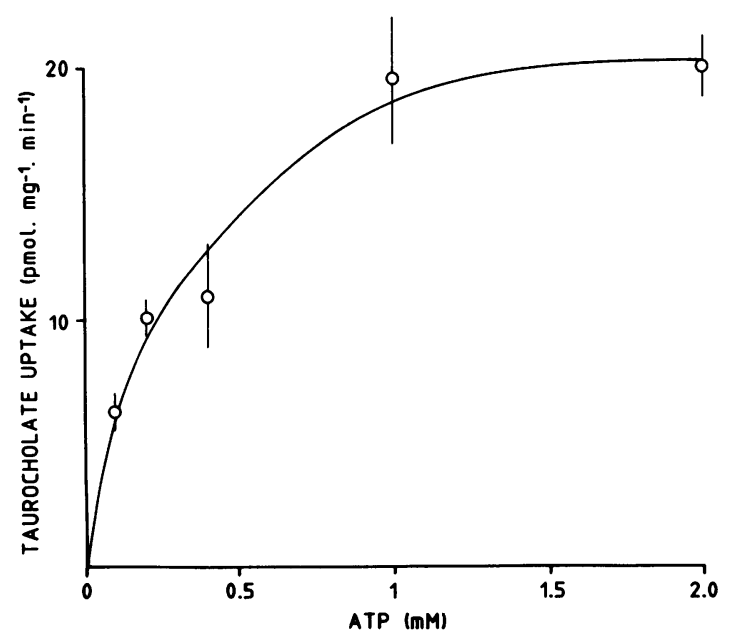

Figure 6. Effect of ATP concentration on TC transport. Initial rapid TC uptake was determined in the presence of $1.0 \mu \mathrm{M}$ TC. Data are mean \pm SD of an experiment in triplicate. Qualitatively similar curves were observed in two additional membrane preparations (see text).
Table I. Effects of Nitrate, Ionophores, DIDS, and DNP-SG on Plasma Membrane ATP-dependent TC Transport

\begin{tabular}{lc}
\hline \multicolumn{2}{c}{ Relative transport } \\
\hline & $\%$ \\
Control & 100 \\
FCCP $(5 \mu \mathrm{M})$ & $81 \pm 9(6)$ \\
Nitrate* & $158 \pm 24(4)$ \\
Valinomycin plus $\mathrm{K}^{+\ddagger}$ & $48 \pm 10(4)$ \\
Valinomycin & $53 \pm 21(3)$ \\
DIDS $(250 \mu \mathrm{M})$ & $6 \pm 5(3)$ \\
DNP-SG $(100 \mu \mathrm{M})$ & $62 \pm 10(4)$ \\
\hline
\end{tabular}

Initial rates of ATP-dependent TC uptake were measured in medium as described in Methods unless specified otherwise. TC concentration was $1.0 \mu \mathrm{M}$ and ATP concentration was $1.0 \mathrm{mM}$. Data are given as percentage of control uptake rate $\pm S E M$, with the number of different membrane preparations used in parentheses. The data presented were obtained from at least one CLPM and one blLPM preparation. No qualitative differences were observed between CLPM and blLPM. * Medium inside as well as outside the vesicles contained $100 \mathrm{mM}$ $\mathrm{KNO}_{3}$ plus $50 \mathrm{mM}$ sucrose instead of $250 \mathrm{mM}$ sucrose in the uptake buffer. ${ }^{\ddagger}$ Medium inside and outside the vesicles contained $100 \mathrm{mM}$ $\mathrm{KCl}$ plus $50 \mathrm{mM}$ sucrose instead of $250 \mathrm{mM}$ sucrose in the uptake buffer; valinomycin concentration was $10 \mu \mathrm{g} / \mathrm{mg}$ protein.

${ }^{\S}$ Medium contained $10 \mu \mathrm{g}$ valinomycin/mg protein.

membrane potential in the presence of $\mathrm{KCl}$, inhibited TC transport, which might point to an involvement of membrane potential in the ATP-dependent transport. However, similar inhibition by valinomycin occurred in the absence of potassium, suggesting a nonspecific effect of valinomycin (Table I).

Inhibitors. DIDS, the classical anion transport inhibitor, strongly inhibited ATP-dependent transport (Table I). DNPSG, which was shown to be transported by an ATP-dependent carrier in rat liver membranes $(13,19,20)$, also inhibited ATPdependent TC transport to a considerable extent (Table I).

\section{Discussion}

Excretion of TC into bile was shown to be a sodium-independent, membrane potential-dependent process in rat liver $(5,8$, 9 ). However, the magnitude of the membrane potential is insufficient to explain the gradient of bile salts across the canalicular membrane. The recent discovery of an ATP-dependent TC excretion system in rat liver may provide a clue for the existence of the extremely high biliary bile salt concentrations (1317). This study clearly demonstrates the presence of an ATPdependent transport system for TC in human liver plasma membranes as well. Transport was temperature dependent and showed a low $K_{\mathrm{m}}$ value $\left(K_{\mathrm{m}}=4.2 \pm 0.7 \mu \mathrm{M}\right.$ and $3.7 \pm 0.5 \mu \mathrm{M}$ for cLPM and blLPM, respectively) for TC. In rat cLPM, $K_{\mathrm{m}}$ values of 47 (14), 26 (15), 7.5 (16), and $2.1 \mu \mathrm{M}$ (17) have been published for the ATP-dependent TC transport system. The $K_{\mathrm{m}}$ for ATP was $0.25 \mathrm{mM}$, which is in the range of values reported in the literature for rat cLPM, i.e., 0.80 (16), 0.67 (15), and $0.064 \mathrm{mM}$ (14), respectively. Experiments using the highly permeable anion nitrate and the protonophore FCCP indicated that neither membrane potential nor $\mathrm{pH}$ gradient was involved in the transport process. Similarly, it has been 
reported that membrane potential is not involved in the ATPdependent TC transport in rat canalicular membranes (14, 17). In our experiments, the ionophore valinomycin did inhibit transport. Since this inhibition was seen in both the absence and the presence of the transferred ion (potassium), this is probably attributable to a nonspecific effect of valinomycin. Similar to observations in rat liver $\operatorname{cLPM}(15,17)$, the anion transport inhibitor DIDS very strongly inhibited ATP-dependent TC transport in human membrane vesicles.

The measured transport rates showed large interindividual variations. There are many factors that may influence the transport rate and may thus be responsible for this variation. First of all there are donor-dependent factors (genetic variations, age, gender, diet, previous medication, etc.). Furthermore, several factors may influence the quality of the isolated liver (quality of the perfusion, preservation time). Finally, the purity of the membranes and their orientation (in rat cLPM, ATP-dependent transport was shown in inside-out vesicles only [15]) may influence the measured transport rate. When corrected for the amount of basolateral membranes present in the membrane fractions, there was an insignificant tendency for an age-dependent decrease in TC transport in the blLPM fraction. It is known that in rat liver ouabain and TC uptake decline upon aging $(35,36)$. It is possible that a significant decrease of TC transport rate only takes place at higher ages than those studied here (maximum age $64 \mathrm{yr}$ ). For other parameters that might influence TC transport (donor gender, preservation time of the liver in UW, and storage time of the membranes in liquid nitrogen), we did not observe clear-cut effects.

ATP-dependent TC transport activity was present in both canalicular and basolateral human plasma membrane preparations, whereas in Sprague-Dawley rats it was demonstrated exclusively $(14,15)$, and in Wistar rats predominantly (16, and our own unpublished results) in canalicular membranes. In the latter rat membrane preparations only low rates of ATP-dependent TC transport were measured in blLPM fractions, possibly due to slight contamination of these fractions with cLPM. As was demonstrated in Results, in the human membranes transport activity in blLPM cannot be explained by the low level of contamination with cLPM. At the moment we do not know whether the presence of the transporter at the human basolateral membrane is physiologically relevant. Several explanations for the appearance of this transporter can be put forward. First, Bartles et al. (37) have shown in rat liver that, after synthesis, several canalicular proteins are first transported to the basolateral side of the cell and subsequently transferred to the canalicular membrane. It is conceivable, therefore, that the transporter is always present in the blLPM, although perhaps in a physiologically nonrelevant concentration. During the preservation of the liver in UW for a prolonged period of time (at least $10 \mathrm{~h}$ in our experiments) redistribution of canalicular and basolateral proteins might take place. Alternatively, the transport of the proteins to the blLPM may be less restrained than the redistribution of the proteins from the blLPM to the cLPM, leading to relatively high concentrations of the transporter at the blLPM. In rats, extrahepatic obstructive cholestasis causes a shift of the bile salt transport system from the canalicular to the basolateral side of the cell (38). The donor livers we studied might, to some extent, be in a "cholestatic state," for instance due to medication of the donor or other factors inherent to the donor procedure. However, this possibility is not very likely, since no clinical signs of cholestasis were observed: plasma bilirubin and alkaline phosphatase were normal. Since we do not know whether TC is the physiological substrate for the ATP-dependent transport observed, it may be of physiological significance to transfer its natural substrate out of the cell and into the blood compartment. For several organic anions, transport from the liver to the blood (sinusoidal efflux) is known to occur (39).

There is some controversy in the literature as to whether in rat liver TC and non-bile acid organic anions share the same canalicular ATP-dependent transporter. In one study (13), ATP-dependent DNP-SG and TC transport showed mutual inhibition. Nishida et al. (15), however, did not observe inhibition of ATP-dependent TC transport by DNP-SG. It was also demonstrated $(15,16)$ that mutant rats lacking ATP-dependent DNP-SG transport retain ATP-dependent TC transport, suggesting different transport mechanisms. In our membrane preparations, DNP-SG, a substrate for the non-bile acid organic anion carrier in rats $(13,19)$, did inhibit ATP-dependent TC transport. This may indicate the use of a common transporter. However, more studies on both rat and human membranes are warranted to investigate the nature and kinetics of this inhibition.

In conclusion, we have demonstrated the presence of a high-affinity, temperature-sensitive, and saturable ATP-dependent TC transport system in human liver plasma membranes. Since this transport is independent of membrane potential and proton gradients, it apparently reflects direct ATPdependent transport.

\section{Acknowledgments}

This work was performed in cooperation with the Groningen Human Liver Cell Research Group. The authors thank Dr. Martin J. Smit for helpful discussions, Albert Gerding and Rick Havinga for technical assistance, and Lodewijk Martijn for drawing the figures.

This research was supported by a grant from the Netherlands Digestive Diseases Foundation (Nederlandse Lever Darm Stichting). F. Kuipers is a Research Fellow of the Royal Netherlands Academy of Arts and Sciences.

\section{References}

1. Wolters, H., M. Spiering, A. Gerding, M. J. H. Slooff, F. Kuipers, M. J. Hardonk, and R. J. Vonk. 1991. Isolation and characterization of canalicular and basolateral plasma membrane fractions from human liver. Biochim. Biophys. Acta. 1069:61-69.

2. Ruifrok, P. G., and D. K. F. Meijer. 1982. Sodium ion-coupled uptake of taurocholate by rat-liver plasma membranes. Liver. 2:28-34.

3. Inoue, M., R. Kinne, T. Tran, and I. M. Arias. 1982. Taurocholate transport by rat liver sinusoidal membrane vesicles:evidence of sodium cotransport. Hepatology. 2:572-579.

4. Duffy, M. C., B. L. Blitzer, and J. L. Boyer. 1983. Direct determination of the driving forces for taurocholate uptake into rat liver plasma membrane vesicles. J. Clin. Invest. 72:1470-1481.

5. Meier, P. J., A. St. Meier-Abt, C. Barrett, and J. L. Boyer. 1984. Mechanisms of taurocholate transport in canalicular and basolateral rat liver plasma membranc vesicles. Evidence for an electrogenic canalicular organic anion carrier. J. Biol. Chem. 259:10614-10622.

6. Meier, P. J. 1988. The transport polarity of hepatocytes. Semin. Liver Dis. 8:293-307.

7. Novak, D. A., F. C. Ryckman, and F. J. Suchy. 1989. Taurocholate transport by basolateral plasma membrane vesicles isolated from human liver. Hepatology. 10:447-453.

8. Inoue, M., R. Kinne, T. Tran, and I. M. Arias. 1984. Taurocholate transport by rat liver canalicular membrane vesicles. Evidence for the presence of an $\mathrm{Na}^{+}$-independent transport system. J. Clin. Invest. 73:659-663. 
9. Meier, P. J., A. S. Meier-Abt, and J. L. Boyer. 1987. Properties of the canalicular bile acid transport system in rat liver. Biochem. J. 242:465-469.

10. Ruetz, S. G. Fricker, G. Hugentobler, K. Winterhalter, G. Kurz, and P. J. Meier. 1987. Isolation and characterization of the putative canalicular bile salt transport system of rat liver. J. Biol. Chem. 262:11324-11330.

11. Ruetz, S., G. Hugentobler, and P. J. Meier. 1988. Functional reconstitution of the canalicular bile salt transport system of rat liver. Proc. Natl. Acad. Sci. USA. 85:6147-6151.

12. Sippel, C. J., M. Ananthanarayanan, and F. J. Suchy. 1990. Isolation and characterization of the canalicular bile acid transport protein of rat liver. Am. J. Physiol. 258:G728-G737.

13. Akerboom, T. P. M., V. Narayanaswami, M. Kunst, and H. Sies. 1991. ATP-dependent S-(2,4-dinitrophenyl)glutathione transport in canalicular plasma membrane vesicles from rat liver. J. Biol. Chem. 266:13147-13152.

14. Adachi, Y., H. Kobayashi, Y. Kurumi, M. Shouji, M. Kitano, and T. Yamamoto. 1991. ATP-dependent taurocholate transport by rat liver canalicular membrane vesicles. Hepatology. 14:655-659.

15. Nishida, T., Z. Gatmaitan, M. Che, and I. M. Arias. 1991. Rat liver canalicular membrane vesicles contain an ATP-dependent bile acid transport system. Proc. Natl. Acad. Sci. USA. 88:6590-6594.

16. Müller, M., T. Ishikawa, U. Berger, C. Klüneman, L. Lucka, A. Schreyer, C. Kannicht, W. Reutter, G. Kurz, and D. Keppler. 1991. ATP-dependent transport of taurocholate across the hepatocyte canalicular membrane mediated by a 110-kDa glycoprotein binding ATP and bile salt. J. Biol. Chem. 266:1892018926.

17. Meier, P. J., B. Stieger, and B. O'Neill. 1991. Evidence for direct ATP-dependent taurocholate transport in isolated canalicular rat liver plasma membrane (cLPM) vesicles. Hepatology. 14:135A. (Abstr.)

18. Nishida, T., Z. Gatmaitan, M. Che, and I. M. Arias. 1991. ATP and membrane potential act synergistically in canalicular bile acid transport. Hepatology. 14:145A. (Abstr.)

19. Kobayashi, K., Y. Sogame, K. Hayashi, P. Nicotera, and S. Orrenius. 1988. ATP stimulates the uptake of S-dinitrophenylglutathione by rat liver plasma membrane vesicles. (Fed. Eur. Biochem. Soc.) FEBS Lett. 240:55-58.

20. Kobayashi, K., Y. Sogame, H. Hara, and K. Hayashi. 1990. Mechanism of glutathione $\mathrm{S}$-conjugate transport in canalicular and basolateral rat liver plasma membranes. J. Biol. Chem. 265:7737-7741.

21. Kobayashi, K., S. Komatsu, T. Nishi, H. Hara, and K. Hayashi. 1991. ATP-dependent transport for glucuronides in canalicular plasma membrane vesicles. Biochem. Biophys. Res. Commun. 176:622-626.

22. Kitamura, T., P. Jansen, C. Hardenbrook, Y. Kamimoto, Z. Gatmaitan, and I. M. Arias. 1990. Defective ATP-dependent bile canalicular transport of organic anions in mutant $\left(\mathrm{TR}^{-}\right)$rats with conjugated hyperbilirubinemia. Proc. Natl. Acad. Sci. USA. 87:3557-3561.

23. Oude Elferink, R. P. J., R. Ottenhof, W. G. M. Liefting, B. Schoemaker, A. K. Groen, and P. L. M. Jansen. 1990. ATP-dependent efflux of GSSG and GS-conjugate from isolated rat hepatocytes. Am. J. Physiol. 258:G699-G706.

24. Ishikawa, T., M. Müller, C. Klünemann, T. Schaub, and D. Keppler. 1990. ATP-dependent primary active transport of cysteinyl leukotrienes across liver canalicular membrane. Role of the ATP-dependent transport system for glutathione S-conjugates. J. Biol. Chem. 265:19279-19286.
25. Zimniak, P., S. A. Ziller, I. Panfil, A. Radominska, H. Wolters, F. Kuipers, R. Sharma, M. Saxena, M. T. Moslen, M. Vore, et al. 1992. Identification of an anion-transport ATPase that catalyzes glutathione conjugate-dependent ATP hydrolysis in canalicular plasma membranes from normal rats and rats with conjugated hyperbilirubinemia (GY mutant). Arch. Biochem. Biophys. 292:534-538.

26. Kamimoto, Y., Z. Gatmaitan, J. Hsu, and I. M. Arias. 1989. The function of $\mathrm{Gpl70}$, the multidrug resistance gene product, in rat liver canalicular membrane vesicles. J. Biol. Chem. 264:11693-11698.

27. Dubin, I. N., and F. B. Johnson. 1954. Chronic idiopathic jaundice with unidentified pigment in liver cells. Medicine (Baltimore). 33:155-197.

28. Cornelius, C. E., I. M. Arias, and B. I. Osburn. 1965. Hepatic pigmentation with photosensitivity: a syndrome in Corriedale sheep resembling DubinJohnson syndrome in man. J. Am. Vet. Med. Assoc. 146:709-713.

29. Jansen, P. L. M., W. H. M. Peters, and W. H. Lamers. 1985. Hereditary chronic conjugated hyperbilirubinaemia in mutant rats caused by defective hepatic anion transport. Hepatology. 5:573-579.

30. Kuipers, F., M. Enserink, R. Havinga, A. B. M. van der Steen, M. J. Hardonk, J. Fevery, and R. J. Vonk. 1988. Separate transport systems for biliary secretion of sulfated and unsulfated bile acids in the rat. J. Clin. Invest. 81:15931599

31. Kalayoglu, M., H. W. Sollinger, R. J. Stratta, A. M. D'Alessandro, R. M. Hoffmann, J. D. Pirsch, and F. O. Belzer. 1988. Extended preservation of the liver for clinical transplantation. Lancet. i:617-619.

32. Scharschmidt, B. F., E. B. Keeffe, N. M. Blankenship, and R. K. Ockner. 1979. Validation of a recording spectrophotometric method for measurement of membrane-associated Mg- and NaK-ATPase activity. J. Lab. Clin. Med. 93:790799.

33. Goldbarg, J. A., and A. M. Rutenberg. 1958. The colorimetric determination of leucine aminopeptidase in urine and serum of normal subjects and patients with cancer and other diseases. Cancer. 11:283-291.

34. Lowry, O. H., N. J. Rosebrough, A. L. Farr, and R. J. Randall. 1951. Protein measurement with the Folin phenol reagent. J. Biol. Chem. 193:265275.

35. Ohta, M., S. Kanai, Y. Sato, and K. Kitani. 1988. Age-dependent decrease in the hepatic uptake and biliary excretion of ouabain in rats. Biochem. Pharmacol. 37:935-942.

36. Ohta, M., and K. Kitani. 1990. Age-dependent decrease in the hepatic uptake of taurocholic acid resembles that for ouabain: a possible role of surface membrane protein mobility. Biochem. Pharmacol. 39:1223-1228.

37. Bartles, J. R., H. M. Feracci, B. Stieger, and A. L. Hubbard. 1987. Biogenesis of the rat hepatocyte plasma membrane in vivo: comparison of the pathways taken by apical and basolateral proteins using subcellular fractionation. J. Cell Biol. 105:1241-1251.

38. Fricker, G., L. Landman, and P. J. Meier. 1989. Extrahepatic obstructive cholestasis reverses the bile salt secretory polarity of rat hepatocytes. J. Clin. Invest. 84:876-885.

39. Meijer, D. K. F., and H. M. J. Nijssen. 1991. Transport of drugs, proteins and drug-protein conjugates. In Research in perfused liver. F. Ballet and R. G. Thurman, editors. John Libbey, London. 165-208. 